\title{
Fungos micorrízicos arbusculares como indicadores da recuperação de áreas degradadas no Nordeste do Brasil ${ }^{1}$
}

\author{
Arbuscular mycorrhizal fungi as indicators of the recovery of degraded areas in \\ northeastern Brazil
}

\author{
Romero Francisco Vieira Carneiro ${ }^{2 *}$, Francisco Marques Cardozo Júnior ${ }^{3}$, Lucimária Farias Pereira $^{4}$, Ademir \\ Sérgio Ferreira Araújo ${ }^{5}$ e Gladstone Alves Silva ${ }^{6}$
}

\begin{abstract}
RESUMO - Avaliaram-se atributos dos fungos micorrízicos arbusculares (FMAs) na região sob desertificação de Gilbués, PI, Brasil, objetivando monitorar áreas sob diferentes níveis de degradação e recuperação do solo. Amostras de solo foram coletadas na camada de 0-0,2 m, durante o período seco de 2009 em quatro áreas pertencentes a um Argissolo, visualmente definidas como: REC - área em recuperação por contenção da erosão e plantio de gramíneas e leguminosas exóticas; DEG- área altamente degradada; IDEG - área moderadamente degradada; $\mathrm{MN}$ - área de vegetação nativa. Foram analisados a colonização radicular, o número mais provável de propágulos infectivos (NMP), índices de diversidade (Shannon-Wiener, diversidade e dominância de Simpson, equitabilidade de Pielou e Margalef) e os atributos químicos do solo $\mathrm{pH}, \mathrm{H}+\mathrm{Al}$, fósforo e matéria orgânica, usados como variáveis explicativas da variabilidade de atributos dos FMAs por meio de análises multivariadas. A colonização radicular e o NMP de propágulos foram superiores em REC. Os índices de Shannon-Wiener, dominância de Simpson e Margalef foram menores na área DEG, demonstrando serem bons indicadores de alterações na comunidade de FMAs em áreas degradadas. Pela análise de agrupamento hierárquico, a área DEG teve maior dissimilaridade em relação às demais. Pela análise por componentes principais, os índices de Shannon-Wiener, Margalef, de Simpson, a dominância de Simpson e os teores de fósforo foram os parâmetros que mais explicaram a variância total.
\end{abstract}

Palavras-chave: Desertificação-controle. Micorriza. Solos-degradação. Qualidade do solo.

\begin{abstract}
Attributes of arbuscular mycorrhizal fungi (AMF) were evaluated in a region in the process of desertification at Gilbués, Piauí, Brazil, with the objective of monitoring areas with different levels of soil degradation and recovery. Soil samples were collected at a depth of 0 to $0.2 \mathrm{~m}$ during the dry season of 2009 in four areas of ultisol, which were visually defined as: REC an area under recovery by erosion contention and the planting of exotic grasses and legumes; DEG- an area with high degradation; IDEG - an area with moderate degradation; MN - an area of native vegetation. The following were analysed: root colonization, the most probable number of infective propagules (MPN), diversity indices (the Shannon-Wiener index, the Simpson diversity and dominance indices, the Pielou equitability index and the Margalef index) and the soil chemical attributes of $\mathrm{pH}, \mathrm{H}+\mathrm{Al}$, phosphorus and organic matter, used as explanatory variables for the variability of the AMF attributes by multivariate analyses. Root colonization and propagule MPN were higher in the REC area. The Shannon-Wiener index, the Simpson dominance index and the Margalef index were lower in the DEG area, showing that they are good indicators of changes in the AMF community in degraded areas. By hierarchical cluster analysis, the DEG area showed higher dissimilarity in relation to the other areas. By principal component analysis, the Shannon-Wiener index, the Margalef index and the Simpson dominance index, and the phosphorus levels were the parameters that best explained the total variation.
\end{abstract}

Key words: Desertification-control. Mycorrhizae. Land degradation. Soil quality.

\footnotetext{
*Autor para correspondência

${ }^{1}$ Recebido para publicação em 11/03/2011; aprovado em 30/04/2012

Parte da Dissertação de Mestrado pelo Programa em Agronomia - Solos e Nutrição de Plantas do campus Professora Cinobelina Elvas/CPCE, Universidade Federal do Piauí /UFPI, defendida pelo segundo autor, pesquisa financiada pelo CNPq e FAPEPI

${ }^{2}$ Campus Professora Cinobelina Elvas/CPCE, Universidade Federal do Piauí/UFPI, Bom Jesus-PI, Brasil, romero@ufpi.edu.br

${ }^{3}$ Colegiado de Ciências Biológicas, Universidade Estadual do Piauí/UESPI, Corrente-PI, Brasil, professorcardozo@yahoo.com.br

${ }^{4}$ Colegiado de Agronomia, Universidade Federal do Piauí/CPCE, Bom Jesus-PI, Brasil, lucimariafarias@hotmail.com

${ }_{5}^{5}$ Departamento de Engenharia Agrícola e Solos/Centro de Ciências Agrárias, Universidade Federal do Piauí, Teresina-PI, Brasil, asfaruaj@yahoo.com.br

${ }^{6}$ Departamento de Micologia, Universidade Federal de Pernambuco/UFPE, Recife-PE, Brasil, gladstonesilva@yahoo.com
} 


\section{INTRODUÇÃO}

Os fungos micorrízicos arbusculares pertencentes ao filo glomeromycota e classe glomeromycetes, são organismos biotróficos obrigatórios que estabelecem relação simbiótica mutualista com raízes de angiospermas, gimnospermas, além de alguns representantes das briófitas e pteridófitas (SOUZA et al., 2010). Atualmente, encontram-se distribuídos em quatro ordens (Archaeosporales, Diversisporales, Glomerales e Paraglomerales), 13 famílias e 19 gêneros (OEHL; SOUZA; SIEVERDING, 2008), totalizando cerca de 220 espécies reconhecidas (GOTO et al., 2010), com pelo menos 50\% já descritas no Brasil.

Os FMAs possuem papel importante na manutenção da diversidade e produtividade dos ecossistemas vegetais terrestres (SOUZA et al., 2010). Em razão do aumento da produtividade da biomassa vegetal que proporcionam, têm incrementado a dinâmica e aporte de nutrientes ao solo via serrapilheira e colaborado com o aumento do dreno de C da atmosfera (LEAKE et al., 2004). Além disso, os FMAs podem ainda determinar o padrão de sucessão de uma comunidade vegetal (RAMOS ZAPATA; ORELLANA; ALLEW., 2006; STÜRMER; SIQUEIRA, 2010). A degradação dos ecossistemas é resultado de fatores diversos que atuam sobre o solo e sua vegetação, alterando suas propriedades físico-químicas e principalmente biológicas, comprometendo o funcionamento dos sistemas simbióticos, tais como as micorrizas arbusculares (LIMA et al., 2007; SIQUEIRA et al., 2007). A baixa resiliência é uma característica marcante de um ecossistema degradado, pois a sua recuperação pode ser lenta ou até mesmo não ocorrer, o que demanda a intervenção antrópica por meio de práticas de recuperação do solo e consequentemente o monitoramento da sua eficácia (LIMA et al., 2007).

Diferentemente da maioria das áreas degradadas do Brasil, além da suscetibilidade aos processos erosivos, o solo da região de Gilbués no Estado do Piauí, é caracterizado por apresentar, nos materiais remanescentes à erosão, elevados teores de pH e P (SILVA; SALVIANO; ANDRADE, 2007), fatores estes que levam frequentemente à inibição dos FMAs (CARNEIRO et al., 2010). Entretanto, os FMAs vêm sendo considerados como parâmetro crítico para uma eficiente recuperação da cobertura vegetal de áreas degradadas, tanto por meio da introdução de plantas inoculadas com isolados exóticos efetivos (POUYUROJAS; SIQUEIRA; SANTOS, 2006) quanto por meio do manejo de comunidades nativas (SCHREINER et al., 2007). Assim, reconhecer atributos das comunidades nativas de FMAs como indicadores de impactados tanto ambientais quanto de práticas conservacionistas do solo, permitirão inferir mais eficientemente sobre a sua diversidade funcional (SCHREINER et al., 2007), utilizá-los como instrumento de medida da qualidade do solo e viabilizar a sua aplicabilidade como um insumo biotecnológico, sobretudo na recuperação de áreas degradadas com potencial para explorações agrícolas sustentáveis.

No entanto, escassas e inconsistentes ainda são as pesquisas sobre a ecofisiologia da microbiota do solo, em especial aquelas que visam interpretar suas interações bióticas e abióticas através de parâmetros reducionistas ou os chamados indicadores ecológicos (MELLONI et al., 2006).

O objetivo deste trabalho foi determinar atributos relacionados aos fungos micorrízicos arbusculares tais como a colonização radicular, o número mais provável de propágulos infectivos, índices de diversidade e sua relação a atributos químicos do solo visando utilizá-los como indicadores em áreas degradadas e em recuperação na região sob desertificação de Gilbués, PI, no Nordeste brasileiro.

\section{MATERIAL E MÉTODOS}

$\mathrm{O}$ estudo foi realizado em área pertencente ao Núcleo de Pesquisas para Recuperação de Áreas Degradadas e Combate à Desertificação (NUPERADE) no município de Gilbués-PI (945'55”S; 4521'00”W), que apresenta clima Aw - tropical segundo Köppen, com temperaturas médias anuais entre 25 e $36{ }^{\circ} \mathrm{C}$, com oscilação de 800 a $1.200 \mathrm{~mm}$ de precipitação anual e período seco definido entre os meses de maio a novembro (SILVA; SALVIANO; ANDRADE, 2007). O estudo foi realizado em setembro de 2009, sendo registrados $12 \mathrm{~mm}$ de precipitação pluviométrica, $29,8^{\circ} \mathrm{C}$ de temperatura média do ar e $37 \%$ de umidade relativa do ar, dados estes gerados da estação meteorológica localizada no NUPERADE.

Em função do grau de cobertura vegetal e manejo implantado para a recuperação do solo, quatro áreas amostrais foram visualmente definidas como: RECdenominada de área em recuperação a partir do ano de 2003 onde foram construídas barragens em nível para contenção da erosão e posteriormente plantio de leguminosas e gramíneas forrageiras, tais como: Crotalaria juncea, Cajanus cajan L., Leucaena leucocephala e Cenchrus ciliares; DEG- área degradada pela erosão, apresentando solo com exposição do horizonte $\mathrm{C}$, e pobre em cobertura vegetal; IDEG- entorno da mata nativa, aqui definida como aquela em processo inicial de degradação também com presença de horizonte $\mathrm{C}$ exposto, entretanto com cobertura vegetal ligeiramente superior a DEG; e MNmata nativa preservada. Características mais detalhadas das áreas amostrais são descritas na Tabela 1.

A unidade pedológica das áreas amostrais está inserida na Bacia Hidrográfica do Rio Parnaíba (microbacia do Sucuruiú), tendo o Argissolo como classe característica em relevo ondulado a suavemente ondulado dentro da 
faixa de declividade de 2-9\% (Tabela 1). Observouse visualmente, que o solo apresentava-se altamente perturbado pelos efeitos da erosão, chegando à exposição do horizonte $\mathrm{C}$, nitidamente verificado especialmente nas áreas definidas como DEG, IDEG e REC, em ordem decrescente de intensidade de exposição.
Em cada área, amostras com cerca de dois kg de solo em cada ponto, foram coletadas da camada de 0-0,2 m de profundidade, totalizando seis pontos amostrais (repetições) definidos aleatoriamente por caminhamento em ziguezague. Cada ponto foi avaliado em réplica, determinando-se a média para a definição do valor observado de cada variável

Tabela 1 - Características de quatro áreas avaliadas na região sob desertificação de Gilbués, PI, sendo: REC- em recuperação sob influência de barragens em nível para contenção da erosão e posterior plantio de gramíneas e leguminosas forrageiras; DEG - área degradada; IDEG - em processo inicial de degradação e MN - Mata nativa preservada

\begin{tabular}{|c|c|c|c|c|c|}
\hline \multirow{2}{*}{ Família: espécie } & \multirow{2}{*}{ Nome vulgar } & \multicolumn{4}{|c|}{ Áreas } \\
\hline & & REC & DEG & IDEG & MN \\
\hline Cyperaceae: Cyperus SP & Tiririca & $\mathrm{X}$ & & & \\
\hline Fabaceae: Cajanus cajan (L) & Guandú & $\mathrm{X}$ & & & \\
\hline Fabaceae: Crotalaria juncea & Crotalária & $X$ & & & \\
\hline Fabaceae: Leucaena leucocephala & Leucena & $\mathrm{X}$ & & & \\
\hline Poaceae: Cenchrus ciliares & Buffel & $\mathrm{X}$ & & & \\
\hline Poaceae: Hyparrheria SP & - & & $X$ & $\mathrm{X}$ & \\
\hline Tiliaceae: Luehea cardiacans & Açoita cavalo & & & $X$ & \\
\hline Malpighiaceae: Byrsonima SP & Murici & & & $X$ & \\
\hline Anacardiaceae: Myracrodruon urundeuva & Aroeira & & & & $\mathrm{X}$ \\
\hline Bignoniaceae: Jacaranda SP & Caroba & & & & $\mathrm{X}$ \\
\hline Sterculiaceae: Guazuma SP & Mutamba & & & & $\mathrm{X}$ \\
\hline Rubiaceae: Alibertia SP & Marmelada & & & & $\mathrm{X}$ \\
\hline Lamiaceae: Ocimum basilicum & Alfavaca & & & & $\mathrm{X}$ \\
\hline Fabaceae: Aeschynomene americana $(\mathrm{L})$ & Angiquim & & & & $\mathrm{X}$ \\
\hline Sapindaceae: Cardiopermum halicacabum (L) & Chumbinho & & & & $\mathrm{X}$ \\
\hline Euphorbiaceae: Croton sincrorensis & Velame & & & & $\mathrm{X}$ \\
\hline Dilleniaceae: Curatella americana $(\mathrm{L})$ & Sambaiba & & & & $\mathrm{X}$ \\
\hline Fabaceae: Bauhinia sp & Miroró & & & & $\mathrm{X}$ \\
\hline Combretaceae: Crombretum duarteanum & Vaqueta & & & & $\mathrm{X}$ \\
\hline Localização das áreas amostrais & Unidades & REC & DEG & IDEG & $\mathrm{MN}$ \\
\hline Longitude (W) & & $45^{\circ} 20^{\prime} 32,2^{\prime \prime}$ & $45^{\circ} 20^{\prime} 29,2^{\prime \prime}$ & $45^{\circ} 20^{\prime} 41,1^{\prime \prime}$ & $45^{\circ} 20^{\prime} 42,7^{\prime \prime}$ \\
\hline Latitude (S) & & $09^{\circ} 52^{\prime} 49,6^{\prime \prime}$ & $09^{\circ} 52^{\prime} 48,3^{\prime \prime}$ & $09^{\circ} 52^{\prime} 33,0^{\prime \prime}$ & $09^{\circ} 52^{\prime} 32,1^{\prime \prime}$ \\
\hline Altitude & $\mathrm{m}$ & 449 & 452 & 460 & 441 \\
\hline Faixa de declividade & $\%$ & $5-9$ & $5-9$ & $5-9$ & $2-5$ \\
\hline \multicolumn{6}{|c|}{ Propriedades químicas e físicas do solo } \\
\hline Matéria Orgânica & dag $\mathrm{kg}^{-1}$ & 0,2 & 0,05 & 0,5 & 1,0 \\
\hline $\mathrm{pH}$ & $\left(\mathrm{em} \mathrm{H}_{2} \mathrm{O}\right)$ & 7,2 & 8,2 & 8,2 & 7,1 \\
\hline Acidez Potencial $(\mathrm{H}+\mathrm{Al})$ & $\mathrm{cmol}_{\mathrm{c}} \mathrm{dm}^{-3}$ & 1,0 & 0,9 & 0,8 & 1,7 \\
\hline P (Mehlich I) & $\mathrm{cmol}_{\mathrm{c}} \mathrm{dm}^{-3}$ & 20,2 & 37,8 & 12,8 & 20,9 \\
\hline Argila & $\mathrm{g} \mathrm{kg}^{-1}$ & 510,0 & 520,1 & 500,4 & 510,2 \\
\hline Silte & $\mathrm{g} \mathrm{kg}^{-1}$ & 100,3 & 90,8 & 100,5 & 90,7 \\
\hline Areia & $\mathrm{g} \mathrm{kg}^{-1}$ & 370,9 & 380,1 & 390,1 & 390,1 \\
\hline Densidade & $\mathrm{g} \mathrm{cm}^{-3}$ & 1,23 & 1,40 & 1,38 & 1,15 \\
\hline
\end{tabular}


por repetição. As espécies vegetais listadas na Tabela 1 foram identificadas a partir de amostragens de folhas e inflorescências coletadas em um raio de um metro de cada ponto amostral, sendo as mesmas fotografadas, prensadas e acondicionadas para identificação junto ao acervo de espécies do herbário Afrânio Fernandes da Universidade Estadual do Piauí. Os atributos físico-químicos foram determinados conforme método da Empresa Brasileira de Pesquisa Agropecuária (1997).

O percentual de colonização micorrízica $(\mathrm{Col})$ foi determinado pela técnica de montagem de lâminas, descrita por Giovannetti e Mosse (1980), em que as raízes coletadas foram diafanizadas e coradas segundo Phillips e Hayman (1970). Posteriormente, foram atribuídas diferentes classes de colonização, pelo agrupamento de amostras segundo Kormanick e McGraw (1982). A avaliação do NMP de propágulos infectivos de FMA foi realizada conforme Feldmann e Idczak (1994). Em casa de vegetação, o solo diluente (correspondente à amostra composta de cada área) foi homogeneizado, peneirado e autoclavado a $121^{\circ} \mathrm{C}$, em dois períodos de duas horas e em dias alternados. As amostras do solo-inóculo para cada área foram homogeneizadas, secas e peneiradas $(4 \mathrm{~mm})$. Em seguida, foram realizadas diluições nas proporções de 0;1:10; 1:100 e 1:1.000 (solo-inóculo:solo-diluente, v:v) e colocadas em copos plásticos de 0,1 L, com cinco repetições, cultivando-se por 30 dias com o painço (Panicum miliaceum L.) como planta teste. O resultado do NMP (propágulos $\mathrm{cm}^{-3} \mathrm{de}$ solo) foi obtido na tabela de Cochran.

Para estimativa dos índices de diversidade, inicialmente as espécies de FMA foram identificadas para a determinação das frequiências absolutas (Tabela 2). Para tanto, os glomerosporos foram extraídos de 0,05 L de solo pelo método de peneiramento úmido (GERDEMANN; NICOLSON, 1963) e centrifugação em água e sacarose. Em seguida, foram transferidos para lâminas de microscopia e montados em PVLG (álcool-polivinilico e lactoglicerol). Com base nas características morfológicas dos glomerosporos, as espécies foram identificadas utilizando-se o manual de Schenk e Pérez (1990) e os sítios eletrônicos das coleções internacionais de FMAs: agro. ar.szczecin.pl/ jblaszkowski e http://invam.caf.wvu.edu (INVAM - International Culture Collection of Arbuscular and Vesicular-Arbuscular Mycorrhizal Fungi), junto ao Laboratório de Micorrizas do Departamento de Biologia da Universidade Federal de Pernambuco.

Posteriormente, a partir do número de indivíduos de cada espécie, os índices de diversidade e dominância de Simpson (Ds, L), Shannon-Wiener (H'), equitabilidade de Pielou ( $\mathbf{J}$ '), foram estimados considerando a abundância

Tabela 2 - Frequências absolutas das espécies de FMAs obtidas em solo da região sob desertificação de Gilbués, PI; em quatro áreas amostrais: REC- em recuperação sob influência de barragens em nível para contenção da erosão e posterior plantio de gramíneas e leguminosas forrageiras; DEG - Degradada; IDEG - Em processo inicial de degradação e MN - Mata nativa preservada

\begin{tabular}{|c|c|c|c|c|}
\hline \multirow{2}{*}{ Espécies de FMA } & \multicolumn{4}{|c|}{ Áreas Amostrais } \\
\hline & REC & DEG & IDEG & $\mathrm{MN}$ \\
\hline Acaulospora bireticulata F.M. Rothwell \& Trappe & $15^{*}$ & & & \\
\hline A. mellea Spain \& N.C. Schenck & 6 & & & 4 \\
\hline A. morrowiae Spain \& N.C. Schenck & 439 & 147 & 187 & 25 \\
\hline A. scrobiculata Trappe & & & 12 & 4 \\
\hline Acaulospora sp.1 & 10 & & 14 & \\
\hline Archaeospora trappei (R.N. Ames \& Linderman) J.B. Morton \& D. Redecker emend. Spain & 13 & & 1 & \\
\hline Glomus constrictum Trappe & 77 & 6 & 24 & 173 \\
\hline G. eburneum L.J. Ken., J.C. Stutz \& J.B. Morton & & & & 2 \\
\hline Glomus sp.1 & 6 & & & 4 \\
\hline Glomus sp.2 & 10 & 25 & & 3 \\
\hline Glomus sp.3 & & & 16 & 20 \\
\hline Glomus sp.4 & & & 7 & \\
\hline Intraspora sp.1 & 10 & & & \\
\hline Scutellospora sp.1 & & & 10 & \\
\hline Número de Espécies por Área & 9 & 3 & 8 & 8 \\
\hline
\end{tabular}

*Representa o valor (ni) observado em $50 \mathrm{~mL}$ de solo e utilizado para determinação dos indicadores de diversidade 
Tabela 3 - Indicadores de diversidade de fungos micorrízicos arbusculares e seus estimadores, utilizados na avaliação de áreas sob influência da desertificação em Gilbués, PI

\begin{tabular}{|c|c|}
\hline Abundância (ni) & $\mathrm{N}^{\mathrm{o}}$ de esporos da espécie i \\
\hline Abundância relativa (AR) & $\begin{array}{c}\mathrm{AR}=\left(\mathrm{N}^{\mathrm{o}} \text { esporos das espécies e/ou gênero } \times 100 \%\right) / \mathrm{N}^{\circ} \text { total de } \\
\text { esporos identificados }\end{array}$ \\
\hline Frequencia isolada $(\mathrm{FI})$ & $\begin{array}{c}\mathrm{FI}=\left(\mathrm{N}^{\mathrm{o}} \text { de amostras de solo onde a espécie e/ou gênero ocorreram } \mathrm{x}\right. \\
\qquad 100 \%) / \mathrm{N}^{\circ} \text { total de amostras }\end{array}$ \\
\hline Índice de diversidade de Shannon-Wiener ( $\left.\mathrm{H}^{\prime}\right)$ & $\mathrm{H}^{\prime}=-\Sigma \mathrm{Pi}^{*} \log \mathrm{Pi}$ \\
\hline Equitabilidade de Pielou (J') & $\mathrm{J}^{\prime}=\mathrm{H}^{\prime} / \mathrm{H}^{\prime} \mathrm{Max}$ \\
\hline İndice de dominância de Simpson (L) & $\mathrm{L}=\Sigma \mathrm{ni}(\mathrm{ni}-1) / \mathrm{N}(\mathrm{N}-1)$ \\
\hline Índice de diversidade de Simpson (Ds) & Ds $=1-L$ \\
\hline
\end{tabular}

*Pi $=$ ni/N,sendo $n i=n^{\circ}$ de esporos da espécie $i ; \mathrm{N}=\mathrm{n}^{\circ}$ total de esporos identificados; $\mathrm{H}^{\prime}$ max $=$ log $\mathbf{s}$, onde $\mathbf{s}=$ total de espécies e/ou gêneros identificados (BROWER e ZAR, 1984)

total (ni), abundância relativa (AR) e frequência isolada (FI), conforme metodologia de Brower e Zar (1984) (Tabela 3). O índice de Margalef (D $\alpha)$ foi calculado com auxílio do software DivEs, optando-se pelo logaritmo de base 10.

Realizou-se análise estatística dos dados, inicialmente pela comparação de médias utilizando-se o respectivo erro padrão, por meio do programa Assistat. Posteriormente, para análise de conjunto complexo de dados simultâneos, procedeu-se o uso da estatística multivariada por meio das técnicas por agrupamento hierárquico (método de Ward utilizando-se a distância euclidiana com padronização z-scores) e análise de componentes principais utilizando o software Statistical Package for the Social Sciences (SPSS) versão 17.0. Para análise dos grupos formados pelo Dendrograma, adotouse um ponto de corte baseado na maior amplitude das distâncias de junção dos grupos hierárquicos formados e, no estudo por componentes principais, reteve-se o número de componentes que explicaram até $70 \%$ da variância acumulada (FERREIRA, 2008).

\section{RESULTADOS E DISCUSSÃO}

As maiores taxas de colonização micorrízica arbuscular (Col) foram encontradas nas raízes extraídas das áreas em recuperação (REC) e em processo inicial de degradação (IDEG). Os menores valores foram verificados na área degradada (DEG) e de mata nativa (MN) (Tabela 4). Para Miranda, Vilela e Miranda. (2005) leguminosas e gramíneas de interesse agronômico, plantas medicinais e xerofíticas sob condições de cultivo, são altamente micotróficas. O cultivo de Crotalaria juncea, Cajanus cajan L. (feijão-guandu), Leucaena leucocephala, Cenchrus ciliares (capim buffel), pode ter contribuído favoravelmente para que na área REC fossem verificados valores superiores em relação às demais áreas, para os percentuais de colonização, bem como o maior número de amostras concentradas na classe de colonização 4 (Tabela 4).

As raízes das plantas da área DEG apresentaram taxa de colonização micorrízica estatisticamente igual à

Tabela 4 - Colonização micorrízica arbuscular (Col) distribuídas por classes e médias gerais, número mais provável (NMP) de propágulos infectivos de FMAs no solo e correlação de Pearson entre Col x NMP na área de desertificação de Gilbués, PI

\begin{tabular}{lcccccccc}
\hline \multirow{2}{*}{ Áreas $^{1}$} & \multicolumn{5}{c}{ Classes $^{2}$ (Colonização) } & Col $(\%)$ & NMP de propágulos cm ${ }^{-3}$ & Correlação de Pearson Col x NMP \\
\cline { 2 - 5 } & 1 & 2 & 3 & 4 & 5 & & 130 & $0,504^{\text {ns }}$ \\
REC & 0 & 1 & 2 & 3 & 0 & $45,7+8,5^{2}$ & 22 & 27 \\
DEG & 0 & 3 & 3 & 0 & 0 & $27,7+4,2^{2}$ & 79 & \\
IDEG & 0 & 1 & 3 & 2 & 0 & $41,0+6,7^{2}$ & 0 & \\
MN & 0 & 3 & 2 & 1 & 0 & $28,5+8,1^{2}$ & 27 & \\
\hline
\end{tabular}

${ }^{1}$ REC: em recuperação sob influência de barragens em nível para contenção da erosão e posterior plantio de gramíneas e leguminosas forrageiras; DEG: Degradada; IDEG: em processo inicial de degradação e MN: mata nativa preservada. ${ }^{2}$ classes de colonização: 1 (0-5\%), 2 (6-25\%), 3 (26-50\%), 4 (51-75\%), 5 (76-100\%). ${ }^{2}$ erro padrão da média para comparações das taxas de colonização entre as áreas avaliadas, ${ }^{n s}$ não significativo 
MN. Notou-se que em DEG, onde havia apenas plantas do gênero Hyparrheria (Tabela 1) a colonização foi 1,48 vezes menor que a verificada em IDEG, onde além do gênero Hyparrheria encontrou-se ainda as espécies açoitacavalo (Luehea cardiacans) e murici (Byrsonima spp).

Nas áreas DEG e MN, verificou-se um maior número de amostras distribuídas na classe de colonização 2. Como as amostras de raízes foram retiradas sem identificação direta da planta de origem, supõe-se que a maior diversidade vegetal em IDEG comparativamente à DEG (Tabela 1), esteja contribuindo para tais resultados. Entretanto, é mais relatado o impacto de fatores como variações do $\mathrm{pH}$, fósforo e água (MIRANDA, VILELA; MIRANDA, 2005), intensidade luminosa sobre o estrato vegetal (ZANGARO et al., 2007), que ao influenciarem a atividade metabólica da planta, afetam a amplitude dos valores de colonização radicular. Assim, era esperado um efeito negativo do maior teor de $\mathrm{P}$ verificado em IDEG (Tabela 1), fato que não foi confirmado e que pode estar relacionado às características das plantas dominantes presentes naquela área.

Guadarrama et al. (2008) destacaram a importância de se verificar a viabilidade dos propágulos nativos, e em floresta tropical mexicana registraram que mais de $40 \%$ do micélio extrarradicular não estava viável, ao que foi atribuída a baixa da infectividade dos FMAs daquele ecossistema. A capacidade de colonizar raízes varia significativamente conforme a espécie de FMA (HART; READER, 2004), e considera-se que a carência de propágulos de FMAs hábeis, pode indicar fragilidade e vulnerabilidade de um ecossistema à degradação. Por outro lado, a área DEG com a presença de um único gênero vegetal (Tabela 1), pode apresentar uma interação FMA-solo-planta considerada adaptada a tais condições (SCHREINER, 2007), conferindo habilidade àquelas plantas para sobrevivência em um ambiente com alto nível de estresse.

Zangaro et al. (2007), em estudo realizado no Sul do Brasil, destacaram que a taxa de colonização micorrízica é dependente do grupo sucessório a que pertence as plantas na comunidade. Para estes autores, plantas pioneiras (estágio sucessório inicial) apresentam maiores taxas de colonização. Nos estágios sucessórios mais avançados as plantas podem se tornar mais independentes da condição micorrízica (SIQUEIRA et al., 2007). Provavelmente, as plantas identificadas em $\mathrm{MN}$, pertencentes a estágios sucessórios mais avançados, apresentem baixa colonização micorrízica nesta área.

Souza et al. (2003) estudando áreas degradadas do semi-árido nordestino associaram menor colonização à maior concentração de fósforo no solo $\left(>40 \mathrm{mg} \mathrm{dm}^{-3}\right)$. Segundo os autores, em áreas degradadas, elevados índices pluviométricos causam exposição do embasamento rochoso sedimentar e perda da fertilidade do solo. Entretanto, condição inversa foi verificada no presente estudo. Em função da riqueza do material de origem do solo em estudo (SILVA; SALVIANO; ANDRADE, 2007), verificaram-se altos valores de $\mathrm{pH}$ e $\mathrm{P}$, que podem influenciar negativamente a colonização de raízes para algumas espécies de FMA, mas ao mesmo tempo pode selecionar espécies de FMA adaptadas a tais condições. Neste contexto, Schreiner (2007) salientou que o isolamento de FMAs nativos para uso como inoculante pode promover benefícios adicionais sobre a planta hospedeira, em relação à FMAs exóticos não adaptados, tais como o aumento na absorção de $\mathrm{S}$ e micronutrientes.

Verificou-se maior número de propágulos infectivos (NMP) de FMAs em REC (Tabela 4). Possivelmente, a presença de diferentes espécies de leguminosas e gramíneas introduzidas, tenha contribuído para aumentar o NMP nessa área. Nas áreas DEG e IDEG foram detectados os menores valores de NMP. Miranda, Vilela e Miranda (2005) salientaram que menores quantidades de propágulos de FMA são encontradas em áreas com vegetação degradada, e que em áreas de cerrado a densidade de propágulos está relacionada com a textura do solo, sendo os mais arenosos com menores densidades. Nas quatro áreas estudadas, verificaram-se maiores valores de NMP em relação aos resultados de Lima et al. (2007) e Souza et al. (2003), que avaliaram áreas degradadas de caatinga. Supõe-se que a característica argilosa e tipo de vegetação da área em estudo (Tabela 1) possam estar contribuindo com os maiores valores encontrados em relação aos autores citados.

Não houve correlação significativa entre NMP e colonização radicular (Tabela 4). Entretanto, Mergulhão et al. (2007) encontraram correlação negativa significativa entre esses dois parâmetros somente em condição satisfatória de umidade no solo para o crescimento das plantas. Para Hart e Reader (2004) o potencial de inóculo de FMA no solo é melhor explicado pela interação FMAtipo de vegetação. O maior valor de NMP encontrado em REC assemelhou-se aos resultados de Ramos-Zapata, Orellana e Allen (2006) que verificaram maior número de propágulos infectivos em áreas com 10 anos de pousio, quando comparado com a vegetação natural próxima o que corrobora a afirmativa de Hart e Reader (2004).

$\mathrm{Na}$ área MN, o valor do NMP foi inferior a REC. Zangaro et al. (2007) encontraram maior potencial de inóculo em fragmentos florestais da Mata Atlântica, onde nos estágios iniciais de sucessão, prevalecem espécies pioneiras eficientes na multiplicação de FMAs. Esses autores afirmaram também, que em estágios sucessivos posteriores (à semelhança do que se verifica em MN), diminuem-se o número de propágulos infectivos de FMAs no solo. 
Pelos resultados de NMP verificados em DEG e IDEG, os distúrbios relacionados à erosão podem estar contribuindo para redução de propágulos infectivos nas áreas com menor diversidade de cobertura vegetal. Presume-se, pelos valores verificados em REC considerando as condições edafoclimáticas do presente estudo, que para se recuperar populações potencialmente infectivas de FMAs em áreas suscetíveis a erosão, seja necessário implantar práticas que incluam o cultivo de espécies vegetais exóticas.

$\mathrm{Na}$ área DEG verificou-se o menor valor para os índices de Shannon-Wiener $\left(\mathrm{H}^{\prime}=0,23\right)$ e de Margalef $(\mathrm{D} \alpha=0,88)$, ambos significativamente diferentes em relação às outras três áreas avaliadas, demonstrando que estes foram índices sensíveis às mudanças que ocorreram neste ecossistema (Tabela 5). Para Brower e Zar (1984), as medidas geradas pelo índice de Margalef ( $\mathrm{D} \alpha)$ não permitem diferenciar a diversidade entre comunidades quando as mesmas apresentam o mesmo número de espécies e de indivíduos. Entretanto, na comparação entre as áreas IDEG e MN, com igual número de espécies e valores aproximados de indivíduos, a mensuração do índice de Margalef, Shannon-Wiener e diversidade de Simpson, apresentaram tendências semelhantes.

Para Brower e Zar (1984) uma comunidade com alta diversidade terá baixa dominância. $\mathrm{Na}$ área DEG houve maior dominância de Simpson $(L=0,70)$ e menor índice de diversidade de Simpson (Ds =0,29). Verificou-se que a elevada abundância $(n i=147)$ e a maior frequência isolada (58\%) da espécie Acaulospora morrowiae nas amostras de DEG, indicaram que há dominância de poucas espécies na comunidade de FMAs nesta área. Wu et al. (2007) afirmaram que o número de espécies de plantas correlacionaram-se positivamente com a abundância de FMA.

A equitabilidade avaliada pelo índice de Pielou (J') foi superior na área IDEG, com valor de 0,56. Sturmer e Siqueira (2010) demonstraram que a substituição vegetal de uma floresta primária na região amazônica para atividades agrícolas não alterou a diversidade de FMAs, e que práticas de manejo cultural contribuem para manter a diversidade em níveis elevados, concordando com os valores verificados em REC no presente estudo.

Associando-se os índices de diversidade anteriormente citados a atributos químicos do solo, pela Análise de Agrupamento Hierárquico, partindo-se do ponto de corte assinalado no Dendrograma (Figura 1), as áreas REC, IDEG e MN foram agrupadas em um mesmo grupo. A área DEG teve maior dissimilaridade em relação às demais áreas permanecendo isolada. Supõese que as práticas de conservação visando à recuperação do solo, neste estudo avaliadas pela diversidade de FMA e atributos químicos do solo, permitiram a inclusão de REC em um mesmo grupo de IDEG e MN.

Pela análise de componentes principais, verificase que os índices de Shannon-Wiener, Margalef, diversidade e dominância de Simpson e os teores de fósforo foram as variáveis mais correlacionadas (Tabela 6) com o componente principal 1, que por sua vez explica $49,3 \%$ da variância total. O segundo componente principal explicou 30,3\% da variância, sendo que os atributos químicos acidez potencial $(\mathrm{H}+\mathrm{Al})$, teor de matéria orgânica e $\mathrm{pH}$ foram os de maior correlação com este componente. Assim, verificouse que apenas atributos químicos apresentaram alta correlação com o componente 2, diferentemente dos atributos relativos aos FMAs que apresentaram alta correlação com o componente 1 (Figura 2). Ratificase assim, a importância da avaliação da diversidade dos FMAs para a indicação do grau de recuperação de áreas em processo de degradação, conforme pode ser verificado na dispersão das variáveis pela Figura 2.

Ressalta-se que uma medida de diversidade tem o objetivo de expressar a complexidade estrutural e ecológica das interações bióticas e abióticas da comunidade microbiana por meio de um único número reducionista (MELLONI et al., 2006). Para estes autores, o

Tabela 5 - Índices de diversidade para as comunidades de FMAs em áreas sob diferentes níveis de degradação na região sob desertificação de Gilbués, PI, sendo: REC - em recuperação sob influência de barragens em nível para contenção da erosão e posterior plantio de gramíneas e leguminosas forrageiras; DEG - altamente degradada; IDEG - em processo inicial de degradação; MN - mata nativa preservada

\begin{tabular}{lccccc}
\hline \multirow{2}{*}{\multicolumn{1}{c}{ Índices de diversidade }} & \multicolumn{2}{c}{ Áreas amostrais } & \multicolumn{2}{c}{ Erro padrão $^{1}$} \\
\cline { 2 - 6 } & REC & DEG & IDEG & MN \\
\hline Índice de Shannon-Wiener (H') & 0,41 & 0,23 & 0,50 & 0,42 & $+0,04$ \\
Equitabilidade de Pielou (J') & 0,43 & 0,49 & 0,56 & 0,47 & $+0,02$ \\
Índice de dominância de Simpson (L) & 0,58 & 0,70 & 0,49 & 0,55 & $+0,03$ \\
Índice de diversidade de Simpson (Ds) & 0,42 & 0,29 & 0,50 & 0,44 & $+0,03$ \\
Índice de Margalef (D $\alpha$ ) & 2,89 & 0,88 & 2,87 & 2,95 & $+0,40$ \\
\hline
\end{tabular}

${ }^{1}$ Para comparações em linha 
Figura 1 - Dendrograma obtido com o Software SPSS v.17 com base nos atributos de FMAs: colonização radicular, NMP, índices de diversidade (Shannon-Wiener, diversidade e dominância de Simpson, equitabilidade de Pielou e Margalef) e nos atributos químicos do solo: pH, H+Al, fósforo e matéria orgânica; para áreas sob diferentes níveis de degradação e em recuperação do solo em Gilbués, PI, sendo: REC = área em recuperação; $\mathrm{DEG}$ = área altamente degradada; $\mathrm{IDEG}$ = área em processo inicial de degradação e $\mathrm{MN}$ = área de mata nativa preservada

Distância reescalonada da associação de grupos

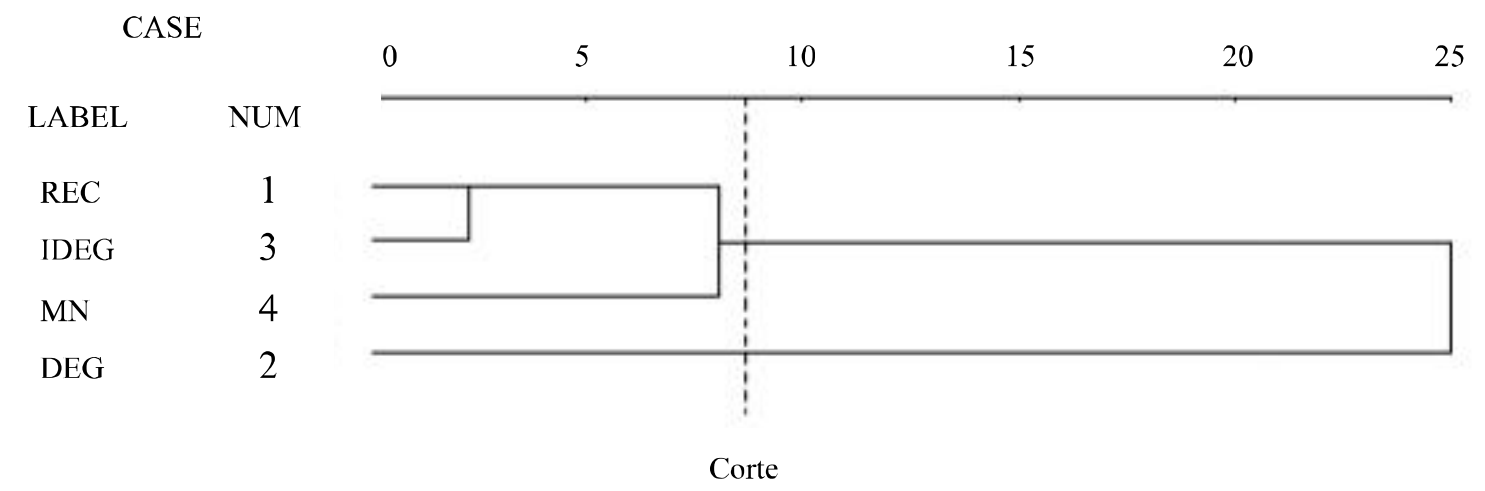

Tabela 6 - Variância explicada e pesos das variáveis, em análise por componentes principais, considerando atributos de FMAs nativos (colonização radicular -Col, número mais provável de propágulos infectivos -NMP, índices de diversidade ShannonWiener $(\mathrm{H})$, diversidade (Ds) e dominância (L) de Simpson, equitabilidade de Pielou (J) e Margalef) e atributos químicos do solo ( $\mathrm{pH}, \mathrm{H}+\mathrm{Al}$, fósforo e matéria orgânica do solo), de áreas sob diferentes níveis de degradação do solo em Gilbués, PI

\begin{tabular}{lcc}
\hline \multirow{2}{*}{ Variáveis } & \multicolumn{2}{c}{ Componentes } \\
\cline { 2 - 3 } & $\mathrm{CP}_{1}$ & $\mathrm{CP}_{2}$ \\
\hline $\mathrm{H}$ & 0,997 & 0,074 \\
$\mathrm{~J}$ & 0,311 & $-0,232$ \\
$\mathrm{~L}$ & $-0,992$ & $-0,101$ \\
$\mathrm{Ds}$ & 0,992 & 0,101 \\
Margalef & 0,901 & 0,258 \\
Col & 0,646 & $-0,631$ \\
NMP & 0,224 & 0,090 \\
$\mathrm{P}$ & $-0,992$ & $-0,094$ \\
M.O & 0,264 & 0,962 \\
H+Al & 0,094 & 0,993 \\
pH & $-0,127$ & $-0,925$ \\
Variância (\%) & 49,3 & 30,3 \\
Variância Acumulada (\%) & 49,3 & 79,6 \\
\hline
\end{tabular}

Método de análise por componentes principais, rotação varimax com normalização Kaiser

índice de Shannon-Wiener $\left(\mathrm{H}^{\prime}\right)$ estimado para populações microbianas em áreas sob efeitos da mineração, foi sensível aos efeitos das diferentes técnicas de reabilitação, em especial aquelas por meio do plantio de feijão-guandu e braquiária, reiterando o potencial de utilização daquele bioindicador nos estudos de qualidade de solos.

Para Caproni et al. (2003) o índice de diversidade de Shannon-Wiener é ideal para se estudar os efeitos das perturbações nos ecossistemas, pois este atribui maior peso às espécies não dominantes, as quais, primeiramente sofrem os efeitos dos impactos ambientais. Além disso, os resultados do presente estudo incluem ainda os índices

Figura 2 - Dispersão das variáveis colonização radicular (Col), número mais provável de propágulos infectivos (NMP), índices de diversidade (Shannon-Wiener $(\mathrm{H})$, diversidade (Ds) e dominância (L) de Simpson, equitabilidade de Pielou (J) e Margalef), pH, H+Al, fósforo e matéria orgânica do solo, e de quatro áreas sob variado nível de degradação do solo em Gilbués, PI, onde: REC - em processo de recuperação, MN - mata nativa, IDEG - em início de degradação e DEG - altamente degradada, em função dos dois primeiros componentes principais com $79,6 \%$ de variância acumulada

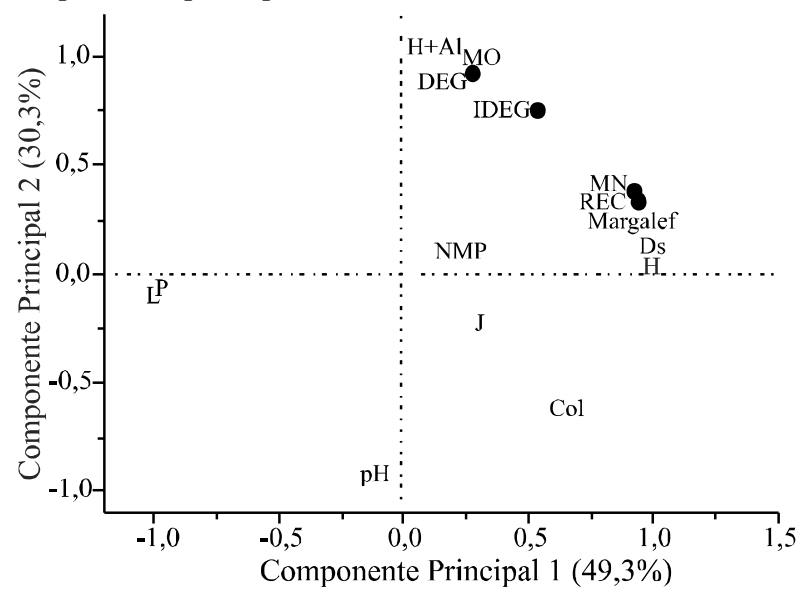


de Margalef $(\mathrm{D} \alpha)$ e diversidade de Simpson (Ds) como indicadores sensíveis da comunidade de FMA relacionados a impactos tanto da recuperação do solo quanto do nível de distúrbio em áreas degradadas.

\section{CONCLUSÕES}

1. Os índices de Shannon-Wiener, dominância de Simpson e Margalef são sensíveis para indicar alterações na diversidade de fungos micorrízicos arbusculares de áreas degradadas;

2. O cultivo de gramíneas e leguminosas forrageiras associadas a práticas de controle da erosão eleva o percentual de colonização das raízes e o número de propágulos infectivos de FMA em áreas degradadas de Gilbués;

3. A análise multivariada com base nas características e diversidade de FMAs nativos e atributos químicos do solo evidencia maior similaridade entre áreas em recuperação e início de degradação com a mata nativa; e maior dissimilaridade destas com área de maior grau de degradação.

\section{REFERÊNCIAS}

BROWER, J. E.; ZAR, J. H. Community similarity. In: BROWER, J. E.; ZAR, J. H. Field \& Laboratory for General Ecology. Dubuque: Win C. Brown Publishers, 1984. p. 161-164.

CAPRONI, A. L. et al. Capacidade infectiva de fungos micorrízicos arbusculares em áreas reflorestadas após mineração de bauxita no Estado do Pará. Pesquisa Agropecuária Brasileira, v. 38, n. 08, p. 937-945, 2003.

CARNEIRO, R. F. V. et al. Doses de fósforo e inoculação micorrízica no cultivo de estilosantes em solo sob condições naturais. Archivos de Zootecnia, v. 59, n. 227, p. 415-426, 2010.

EMPRESA BRASILEIRA DE PESQUISA AGROPECUÁRIA . Centro Nacional de Pesquisas de Solos. Manual de métodos de análises de solos. 2. ed. Rio de Janeiro, 1997.

FELDMANN, F.; IDCZAK, E. Inoculum production of vesiculararbuscular mycorrhizal fungi for use in tropical nurseries. In: NORRIS, J. R.; READ, D. J.; VARMA, A. K. Techniques for mycorrhizal research methods in microbiology. London: Academic Press, 1994. p. 799-817.

FERREIRA, D. F. Estatística multivariada. Lavras: Editora UFLA, 2008. $662 \mathrm{p}$.

GIOVANETTI, M.; MOSSE, B. Evaluation of techniques for measuring vesicular arbuscular mycorrhizal infection in roots. New Phytologist, v. 84, n. 03, p. 489-500, 1980.

GERDEMANN, J. W.; NICOLSON, T. H. Spores of mycorrhizal fungi isolated from soil by wet sieving and decanting. Transactions British Mycological Society, v. 46, n. 02, p. 235-244, 1963.
GOTO, B. T. et al. Checklist of the arbuscular mycorrhizal fungi (Glomeromycota) in the Brazilian semiarid. Mycotaxon, v. 113, p. 251-254, 2010.

GUADARRAMA, P. et al. Propagules of arbuscular mycorrhizal fungi in a secondary dry forest of Oaxaca, Mexico. Revista de Biologia Tropical, v. 56, n. 01, p. 269-277, 2008.

HART, M. M.; READER, R. J. Do arbuscular mycorrhizal fungi recover from soil disturbance differently? Tropical Ecology, v. 45, n. 01, p. 97-111, 2004.

KORMANICK, P. P.; MCGRAW, A. C. Quantification of vesicular-arbuscular mycorrhizae in plant roots. In: SCHENCK, N. C. Methods and principles of mycorrhizal research. Minnesota: American Phytopathological Society, 1982. p. 34-37.

LEAKE, J. R. et al. Networks of power and influence: the role of mycorrhizal mycelium in controlling plant communities and agroecosystem functioning. Canadian Journal of Botany, v. 82, n. 08, p. 1016-1045, 2004.

LIMA, R. L. F. A. et al. Propágulos de fungos micorrízicos arbusculares em solos deficientes em fósforo sob diferentes usos, da região semi-árida no nordeste do Brasil. Revista Brasileira de Ciência do Solo, v. 31, n. 02, p. 257-268, 2007.

MELLONI, R. et al. Eficiência e diversidade fenotípica de bactérias diazotróficas que nodulam caupi (Vigna unguiculata $\mathrm{L}$. Walp) e feijoeiro (Phaseolus vulgaris L.) em solos de mineração de bauxita em reabilitação. Revista Brasileira de Ciência do Solo, v. 30, n. 02, p. 235-246, 2006.

MERGUlhão, A. C. E. S. et al. Potencial de infectividade de fungos micorrízicos arbusculares em áreas nativas e impactadas por mineração gesseira no semi-árido brasileiro. Hoehnea, v. 34, n. 03, p. 341-348, 2007.

MIRANDA, J. C. C.; VILELA L.; MIRANDA, L. N. Dinâmica e contribuição da micorriza arbuscular em sistemas de produção com rotação de culturas. Pesquisa Agropecuária Brasileira, v. 40, n. 10, p. 1005-1014, 2005.

OEHL, F.; SOUZA, F.; SIEVERDING, E. Revision of Scutellospora and description of five new genera and three new families in the arbuscular mycorrhiza forming Glomeromycetes. Mycotaxon, v. 106, p. 311-360, 2008.

PHILLIPS, J. M.; HAYMAN, D. S. Improved procedures for clearing roots and stainind parasitc and vesicular-arbuscular mycorrhyzal funi for rapid assessment of infection. Transactions of the British Mycological Society, v. 55, n. 01, p. 158-161, 1970.

POUYU-ROJAS, E.; SIQUEIRA, J. O.; SANTOS, J. G. D. Compatibilidade simbiótica de fungos micorrízicos arbusculares com espécies arbóreas tropicais. Revista Brasileira de Ciência do Solo, v. 30, n. 03, p. 413-424, 2006.

RAMOS-ZAPATA, J. A., ORELlANA, R.; ALLEN, E. B. Establisment of Desmoncus orthacanthos Martius (Aracaceae): effect of inoculation with arbuscular mycorrhizae. Revista de Biologia Tropical, v. 54, p. 65-72, 2006.

SCHENCK, N. C.; PÉREZ, Y. Manual for the identification of VA- mycorrhizal fungi. 3. ed. Gainesville: Synergistics Publications, 1990. 250 p. 
SCHREINER, R. P. Effects of native and nonnative arbuscular mycorrhizal fungi on growth and nutrient uptake of 'Pinot noir' (Vitis vinifera $\mathrm{L}$.) in two soils with contrasting levels of phosphorus. Applied Soil Ecology, v. 36, n. 02, p. 205-215, 2007.

SILVA, F. B.; SALVIANO, A. A. C.; ANDRADE, J. B. Áreas degradadas em microbacia de Gilbués-PI utilizando imagens do sensor CCD-CBERS-2. In: SIMPÓSIO BRASILEIRO DE SENSORIAMENTO REMOTO, 13., 2007. Florianópolis. Anais... Florianópolis: INPE, 2007. p. 4257-4260.

SIQUEIRA, J. O. et al. Micorrizas e degradação do solo: caracterização, efeitos e ação recuperadora. In: CERETTA, C. A; SILVA, L. S.; REICHERT, J. M. Tópicos em ciência do solo. Viçosa: SBCS, 2007. p. 219-306.

SOUZA, F. A. et al. Classificação e taxonomia de fungos micorrízicos arbusculares e sua diversidade e ocorrência no Brasil. In: SIQUEIRA, J. O. et al.Micorrizas: 30 anos de pesquisa no Brasil. Lavras: Editora UFLA, 2010. p. 15-73.
SOUZA, R. G. et al. Diversidade e potencial de infectividade de fungos micorrízicos arbusculares em área de caatinga, na Região de Xingó, Estado de Alagoas, Brasil. Revista Brasileira de Botânica, v. 26, n. 01, p. 49-60, 2003.

STÜRMER, S. L.; SIQUEIRA, J. O. Species richness and spore abundance of arbuscular mycorrhizal fungi across distinct land uses in Western BrazilianAmazon. Mycorrhiza, 2010. Disponível em: <http://www.springerlink.com/content/0803325748422554/. Acesso em: $11 \mathrm{~m}$,ar. 2011

WU, B. et al. Community structure of arbuscular mycorrhizal fungi in a primary successional volcanic desert on the southeast slope of Mount Fuji. Mycorrhiza, v. 17, n. 16, p. 495-506, 2007.

ZANGARO, W. et al. Root mycorrhizal colonization and plant responsiveness are related to root plasticity, soil fertility and successional status of native woody species in southern Brazil. Journal of Tropical Ecology, v. 23, p. 53-62, 2007. 